\title{
LA INTERDISCIPLINARIEDAD EN LA FORMACION DEL EGRESADO Y EGRESADA DE LA UNIVERSIDAD DE PUERTO RICO: UNA MIRADA DESDE LA DISCIPLINA DEL TRABAJO SOCIAL HACIA OTRAS DISCIPLINAS
}

\author{
Ana Maritza Martínez, Vizcarrondo ${ }^{1}$
}

\section{Resumen}

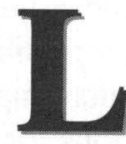

a complejidad social, los retos y desafíos con los que las trabajadoras sociales y los trabajadores sociales nos estamos enfrentando en este siglo XXI, sobre todo en los centros urbanos del país, nos llevan a pensar y a evaluar las nuevas tendencias en la intervención profesional. Ante las transformaciones sociales de tanto impacto en la vida de los seres humanos se sugiere la búsqueda de soluciones integrales para los problemas. Se plantea la interdisciplinaridad como una forma de cooperación entre disciplinas nos ayuda a trascender la fragmentación del saber a través de la especialización, no oponiéndonos a ésta, sino armonizando varias especializaciones (disciplinas) que nos permita abordar la complejidad de los problemas, de las necesidades sentidas y encontrar mejores soluciones cubriendo los vacíos que dejan la especialización y la limitada participación de profesionales de algunas disciplinas en su compromiso por transformar el orden existente. Los enfoques mono disciplinarios y la especialización, están perdiendo terreno frente a formas de trabajo donde la organización rígida es remplazada

\footnotetext{
${ }^{1}$ Catedrática Asociada en el Departamento de Trabajo Social, Facultad de Ciencias Sociales, Universidad de Puerto Rico, Río Piedras.
} 
por colaboraciones, coaliciones donde el poder centralizado es remplazado por el apoderamiento de los participantes y donde las jerarquías de poder ceden su espacio a estructuras horizontales.

La autora comparte su experiencia con prácticas profesionales estudiantiles en el Proyecto CAUCE, adscrito al Recinto de Río Piedras de la Universidad de Puerto Rico, contextualizadas en un Modelo de Colaboración Interdisciplinaria, del cual se explican sus cinco componentes; interdependencia, actividades de nueva creación, metas propias del colectivo y reflexión sobre el proceso.

Descriptores: prácticas interdisciplinarias, colaboración, equipos de trabajos interdisciplinarios, prácticas profesionales estudiantiles, trabajo social comunitario, centros urbanos.

\section{Abstract}

The social complexity and challenges which the social workers are facing in this century XXI, mainly in the urban centers of the country, take us to think and to evaluate the new tendencies in the professional intervention. Faced with the social transformations of as much impact in the life of the human beings, the search of integral solutions for the problems is suggested. The interdisciplinary collaboration considers as a form of cooperation between disciplines helps us to extend the fragmentation of the knowledge through the specialization, not opposing us to this one, but harmonizing several specializations (disciplines). This allowed us to approach the complexity of the problems, the felt needs and to find better solutions covering the emptiness that result from specialization and the limited participation of professionals of some disciplines in their commitment to transform the existing order. The monodisciplinary approaches and the specialization, are withdrawing in front of work forms where the rigid organization is replaced by collaborations, coalitions where the centralized power is replaced by the empowering of the participants and where the hierarchies of being able yield their space to horizontal structures. 
The author shares her experience with Student Professional Practices in the Project CAUCE, assigned to the Río Piedras Campus of the University of Puerto Rico, within the context of a Model for Interdisciplinary Collaboration, of which their five components; interdependence, newly created professional activities, flexibility, collective ownership of goals, and reflection on the process, are explained.

Key words: interdisciplinary practices, collaboration, interdisciplinary work teams, student field practicum, community social work, urban centers.

\section{Introducción}

Aunque la participación de trabajadoras y trabajadores sociales en equipos de diferentes disciplinas data de muchos años, poco se ha estudiado y documentado sobre la práctica interdisciplinaria en trabajo social. El interés sobre este tema nace de mi participación durante tres años (2003 a 2006) en un proyecto autónomo de colaboración interdisciplinaria entre el Recinto de Río Piedras de la Universidad de Puerto Rico y el casco urbano de la comunidad de Río Piedras amparado bajo la Ley Especial para la Rehabilitación de Río Piedras, Ley Núm. 75 del 5 de julio de 1995, según enmendada. El propósito primordial de esta Ley 75 es atender el deterioro físico y social del centro urbano tradicional de Río Piedras. La elaboración del Plan Integral para rehabilitar a Río Piedras tiene como retos de la planificación social: la desigualdad social, deterioro social, desintegración, marginación social, búsqueda de políticas públicas, programas y medidas de intervención, junto con la búsqueda de los factores de peso en la intervención, la identificación de la intervención de la comunidad. La sede de este proyecto es el Centro de Acción Urbana, Comunitaria y Empresarial (CAUCE), donde primero colaboré como supervisora de estudiantes en el curso de Instrucción Práctica en Trabajo Social y luego como coordinadora del componente de prácticas profesionales de estudiantes de diversas disciplinas del Recinto Universitario de Río Piedras de la Universidad de Puerto Rico. 
Las prácticas profesionales son un espacio vital de adiestramiento profesional para estudiantes de diferentes disciplinas del Recinto, éstas se constituyen en un puente de gran relevancia, en el contexto universidad - sociedad. Las estudiantes y los estudiantes participantes en el proyecto, se benefician con experiencias formativas de investigación - acción de carácter interdisciplinario único e innovador. Estudiantes, profesores y profesoras de diversas disciplinas han participado de esta innovadora experiencia académica. Entre estas se encuentran: trabajo social, planificación social, psicología social comunitaria, psicología escolar, geografía, consejería en rehabilitación vocacional, ciencias y tecnología de la información, derecho, educación, y arquitectura. Es a través de los grupos de estudiantes y del peritaje de sus profesores y profesoras participantes en este proyecto, que la Universidad cumple con tres (3) de sus objetivos primordiales relacionados con el servicio comunitario:

1) Fomentar el servicio público y la responsabilidad social con los estudiantes, las estudiantes, docentes, investigadores, investigadoras y personal de apoyo a través de proyectos de servicios, iniciativas curriculares e integración de objetivos al efecto en los prontuarios de cursos, entre otros.

2) Apoyar proyectos comunitarios, cívicos y universitarios, especialmente, la rehabilitación de los centros urbanos y de las comunidades marginadas y vulnerables.

3) Proveer espacios en los currículos universitarios para la práctica e internados en comunidad.

Esta enriquecedora experiencia académica es la que sirve como punto de partida para el presente ensayo, cuyo propósito es aportar al conocimiento en torno a la práctica de trabajo social en equipos de trabajo interdisciplinarios, contextualizando la misma en un modelo de colaboración interdisciplinaria (Bronstein, 2003) aplicado en una comunidad aledaña al Recinto de Río Piedras de la Universidad de Puerto Rico, que puede facilitar y desarrollar aún más este ejercicio práctico dentro del ámbito universitario en general. 


\section{Marco conceptual}

A partir de los años 70, los conceptos de interdisciplinariedad, multidisciplinariedad y transdisciplinariedad han estado presentes en los principales congresos, foros, conferencias y propuestas de investigación y de servicios. Ander- Egg (1999), plantea que el concepto de interdisciplinariedad fue utilizado en 1937 por Louis Writz, pero no fue hasta los años sesenta que el mismo se generalizó. Calderón, Núñez \& Serrano (2003) plantean la dificultad que existe al definir el concepto ya que el término interdisciplinariedad se utiliza constantemente como sinónimo de multidisciplinariedad. La multidisciplinariedad se refiere al trabajo conjunto o compartido de profesionales de disciplinas distintas, mientras que la interdisciplinariedad por su parte implica además una integración de esa actividad y sus resultados a base de una división del trabajo más o menos explícita. La multidisci-plinariedad es la suma de conocimientos de diferentes disciplinas científicas a un interés, necesidad o problema dado, y la interdisciplinariedad, integra y aplica estos conocimientos en su aplicación a ese interés, necesidad o problema. Para que pueda darse la interdisciplinariedad tiene que haber multidisci-plinariedad, es decir unirse distintas disciplinas para explicar y dar solución a un fenómeno, no obstante y de acuerdo a Martín Chacón y Martínez (citado en Calderón, Núñez y Serrano, 2003), lo multidisciplinario no tiene que necesariamente ser interdisciplinario si no aporta a que los conocimientos se integren de una forma más completa que ayude a entender y a tratar mejor las diferentes dimensiones de un fenómeno. Añade Ander - Egg (1999) que el enfoque multidisciplinario combina diferentes disciplinas, pero prevalecen los aspectos individuales de cada disciplina, mientras que la transdisciplinariedad es un concepto nuevo que trasciende los enfoques de las disciplinas que la originan.

Coincido con Calderón, Núñez \& Serrano (2003) al concluir que no todos los problemas necesitan un acercamiento interdisciplinario ya que la naturaleza del mismo no lo requiere, de manera que el tratarlos interdisciplinariamente no aportaría a su comprensión e intervención, "este podría ser el caso del estudio de la medición de los rayos solares ultravioletas en diferentes épocas de año donde un especialista 
de la física podría obtener los datos por sí solo usando los instrumentos adecuados" (p. 153).

En sus consideraciones histórico - ideológicas y metodológicas sobre la interdisciplinariedad, Piaget, Mackenzie y Lazarsfeld nos revelan de forma muy interesante, que consideran la interdisciplinariedad como más difícil de establecer en las ciencias humanas que en las ciencias naturales (según citado en Calderón, Núñez \& Serrano, 2003), admitiendo un orden jerárquico entre estas. Piaget, Mackensie y Lazarsfeld añaden que no se ha resuelto el problema central de la sociología, el cual es:

...ver la sociedad como una totalidad y esclarecer las relaciones entre los subsistemas y el sistema de conjunto. Cada disciplina emplea parámetros que son variables estratégicas para otras disciplinas, lo que abre un vasto campo de investigación a las colaboraciones interdisciplinarias; pero, como no se dispone de una descomposición lineal del sistema en subsistemas, las colaboraciones se reducen demasiado a menudo a meras yuxtaposiciones (según citado en Calderón, Núñez \& Serrano, 2003, p. 156).

Los retos y desafíos con los que trabajadoras y trabajadores sociales nos estamos enfrentando en este siglo XXI, sobre todo en los centros urbanos del país, nos llevan a pensar y a evaluar las nuevas tendencias en la intervención profesional. La multidisciplinariedad y la interdisciplinariedad son los conceptos que fundamentan $\mathrm{y}$ conceptualizan la respuesta a esos retos y desafíos. Cada día, las disciplinas que enmarcan las diferentes profesiones de ayuda, validan la necesidad de unirse con otras disciplinas en su compromiso por solucionar los problemas, atender necesidades básicas, transformar el orden existente.

\section{Justificación y pertinencia de las prácticas profesionales interdisciplinaria}

Existe la gran necesidad entre estudiantes próximos a egresar del sistema universitario (especialmente en la Universidad del Estado), de 
que participen en experiencias educativas y formativas que los ubiquen, en lo que la Organización de las Naciones Unidas para la Educación, la Ciencia y la Cultura (United Nations Educational, Scientific and Cultural Organization, 2000) ha denominado 'profesionales de la ciudad' en el ámbito del Programa de Gestión de Transformaciones Sociales (MOST). De acuerdo a ésta organización, este nuevo proyecto de carácter internacional persigue mejorar la generación de los nuevos profesionales provenientes del sector de la Ciencias Sociales y de la Conducta Humana, el uso de la técnica y del conocimiento, así como fortalecer las capacidades institucionales, para lograr el desarrollo de mejores respuestas ante las nuevas condiciones de adecuación y de gobernabilidad del espacio urbano, tales como por ejemplo, el trabajo en equipo, entre los diferentes sectores y profesionales implicados en este ámbito de trabajo.

La multi-disciplinariedad, la interdisciplinariedad y la transdisciplinariedad abren la posibilidad a explicaciones más integrales u holísticas al cubrir los vacíos que dejan la especialización y la limitada participación de profesionales de algunas disciplinas. Es importante aclarar que estos enfoques no sustituyen ni se oponen al enfoque disciplinario ya que las perspectivas disciplinarias rigurosas e incluso, altamente especializadas, son necesarias. Ante las transformaciones sociales de tanto impacto en la vida de los seres humanos se sugiere la búsqueda de soluciones holísticas para los problemas, la interdisciplinariedad debe ser vista como una forma de cooperación entre disciplinas que permite abordar la complejidad de los problemas, de las necesidades sentidas y encontrar mejores soluciones.

Parker (2005) señala que los enfoques dominados por el individualismo, los enfoques monodisciplinarios y la especialización, están perdiendo terreno frente a formas de trabajo donde la organización rígida es remplazada por colaboraciones, coaliciones donde el poder centralizado es remplazado por el empoderamiento de los participantes y donde las jerarquías de poder ceden su espacio a estructuras horizontales. Para Ander-Egg (1999), la noción de interdisciplinariedad es relativamente fácil de formular si nos guiamos por el "qué" (¿qué queremos hacer?), generalmente 
entendido como la búsqueda por superar el análisis fragmentado; obtenemos así una comprensión más integral de los fenómenos. El problema resulta mayor cuando nos planteamos el "cómo-saber" trabajar de una manera interdisciplinaria. La siguiente figura presenta una formulación esquemática de los elementos constitutivos del trabajo interdisciplinario.

\section{Figura 1}

\section{Esquema interdisciplinario para abordar problemas y fenómenos de estudio}

\begin{tabular}{|l|l|}
\hline ¿Qué queremos hacer? & $\begin{array}{l}\text { ¿Cómo lograr la } \\
\text { interdisciplinariedad? } \\
\text { Con una actitud intelectual }\end{array}$ \\
$\begin{array}{l}\text { Ir más allá del análisis } \\
\text { fragmentado, hacia un } \\
\text { entendimiento amplio } \\
\text { del fenómeno }\end{array}$ & $\begin{array}{l}\text { dirigida hacia la } \\
\text { interdisciplina y al desarrollo } \\
\text { de estrategias de trabajo } \\
\text { colectivo }\end{array}$ \\
\hline \multicolumn{2}{|c|}{ Interdisciplinariedad } \\
\hline $\begin{array}{l}\text { Fomentar una visión } \\
\text { holística en la búsqueda } \\
\text { de soluciones para los } \\
\text { problemas del desarrollo }\end{array}$ & $\begin{array}{l}\text { Incentivar el compartir } \\
\text { diversas perspectivas en } \\
\text { temas de investigación } \\
\text { comunes }\end{array}$ \\
\hline
\end{tabular}

\section{La práctica interdisciplinaria}

En torno a la práctica interdisciplinaria, Romero Sánchez $(2005)^{2}$ plantea que la interdisciplinaridad como práctica es el revaluar la forma de trabajo que implica cada disciplina. La disciplina es un comportamiento de distanciamiento del mundo y del día-a-día, un retiro (discedere), justamente para poder desprenderse de influencias

\footnotetext{
${ }^{2}$ Abogada, especialista en Derecho Administrativo y Docencia Universitaria de la Universidad del Rosario.
} 
perturbadoras y poder discernir (discernere) mejor; quien conseguía hacer esto podía estudiar y entender la realidad de forma más esclarecida, era el discípulo (discens) - supone dependencia de un maestro o guía que muestra el camino (Romero Sánchez, 2005). La práctica interdisciplinaria es una invitación a continuar con los análisis pero manteniendo el contexto y condiciones reales 'sin desmembrarlos y desconocerlos'; además de que ayuda a trascender la fragmentación del saber a través de la especialización, no oponiéndonos a ésta, sino armonizando varias especializaciones (disciplinas) para la comprensión y solución de un interés, una necesidad ó un problema (Romero Sánchez, 2005).

\section{Principios de la interdisciplinaridad}

Evandro Hagáis, en un seminario para profesores impartido en el Departamento de Filosofía de la Universidad de Navarra y como parte de un proyecto de investigación titulado Interdisciplinaridad desde la filosofía de la ciencia, afirmaba los siguientes principios sobre lo que no es la Interdisciplinaridad (según citado en Romero Sánchez, 2005):

- La interdisciplinaridad no es un discurso genérico, ni el desconocimiento de las disciplinas, tampoco es la infra valoración o sobre valoración de disciplinas, o simplemente poner en contacto los discursos de diferentes disciplinas.

- La interdisciplinaridad requiere disciplinas y cada disciplina ofrece conocimientos objetivos y específicos con validez intrínseca.

- En la práctica interdisciplinaria, el contacto con la realidad micro y macro - es inevitable y VITAL.

- La interdisciplinaridad como práctica supone un retorno a esas mismas perturbadoras realidades, y esta vez sin un maestro o guía que determine el camino correcto.

- La autoridad del orientador es sustituida por la visión crítica compartida del equipo interdisciplinario. 


\section{Un Modelo de Colaboración Interdisciplinaria}

Berg-Weger \& Schneider definen colaboración interdisciplinaria como " un proceso interpersonal mediante el cual miembros de diferentes disciplinas contribuyen al logro de una meta en común " (según citado en Bronstein, 2003, p. 698). La colaboración interdisciplinaria es un proceso que va más allá de otros procesos interpersonales relacionados como lo son: la cooperación, comunicación, coordinación y el compañerismo. El Modelo de Colaboración Interdisciplinaria de Bronstein (2003) se enmarca en cuatro enfoques teóricos: (1) la teoría de colaboración multidisciplinaria; (2) la teoría de roles; (3) la perspectiva sistémica ecológica y (4) un modelo de integración de servicios. Bronstein (2003) también identificó, de forma constante en la literatura de la práctica en trabajo social, varios componentes de colaboración interdisciplinaria. Aún con las diferencias existentes entre las disciplinas, este modelo representa de forma genérica los componentes de una colaboración óptima entre trabajadores y trabajadoras sociales y otros profesionales.

Las tareas relacionadas a la colaboración interdisciplinaria de diferentes disciplinas se enmarcan en cinco componentes básicos: interdependencia, actividades de nueva creación profesional, flexibilidad, metas propias del colectivo y reflexión sobre el proceso. Para Bronstein (2003), la 'interdependencia' se refiere a las interacciones interpersonales que dependen de otros para que se puedan lograr las metas $y$ tareas. Para trabajar interdependientemente, cada profesional debe tener un claro entendimiento tanto de sus roles como de los otros profesionales y el uso de éstos adecuadamente. Caracteriza la interdependencia el tiempo formal e informal que se comparte junto; la comunicación oral y escrita entre los colegas profesionales y el respeto hacia las opiniones e insumo de los colegas y las colegas profesionales. En la literatura se hace especial referencia al concepto "equipo de trabajo". Un equipo de trabajo consiste de dos ó más profesionales trabajando unidos. Bronstein (2003) identifica dos patrones que contrastan el concepto de equipo de trabajo. El equipo de trabajo coordinado, caracterizado por distintos roles profesionales, un liderato del equipo designado, la toma de decisiones no se da por consenso y existe poco 
énfasis en el proceso grupal. El equipo de trabajo integrativo, armoniza más con el concepto de colaboración que define este modelo, se caracteriza por creer que la ejecución de las tareas de cada miembro del grupo va a depender de las habilidades y capacidades de cada quien. Una ventaja que frecuentemente enfatiza la literatura relacionada con este concepto es que el peritaje y el conocimiento de diferentes disciplinas maximizan la creatividad para el manejo de los complejos problemas de la actualidad.

Las 'actividades de nueva creación profesional' se refieren a las acciones de colaboración, programas y estructuras que pueden lograr y alcanzar más de lo que pudieran los mismos profesionales si actuaran de forma individual. Estas actividades maximizan el peritaje de cada colaborador en la creación de nuevas estructuras de servicios $\mathrm{y}$ proyectos que afectan de forma significativa a participantes, programas, políticas y la burocracia organizacional.

El componente de 'flexibilidad' se extiende más allá de la interdependencia. Lo caracteriza un sentido de compromiso en casos de desacuerdo y alteración de los roles a los cuales como profesionales fuimos llamados. Bronstein (2003) nos ofrece como ejemplo la situación en un Hospicio donde trabajadores y trabajadoras sociales demuestran flexibilidad al utilizar el conocimiento obtenido en su participación en el equipo interdisciplinario de trabajo con enfermeras y médicos, para contestar preguntas sencillas sobre el cuidado médico a pacientes. Cómo componente en un modelo de colaboración la flexibilidad demanda relaciones menos jerárquicas.

Las 'metas propias del colectivo' se refieren a la responsabilidad compartida durante todo el proceso para el logro de las metas, incluyendo su diseño, definición, desarrollo y logro en conjunto de las metas. Esto incluye un compromiso enfocado al cuidado y bienestar de los sistemas participantes por profesionales de diferentes disciplinas, las y los participantes unidas y unidos en un proceso activo para el logro de las metas propuestas. Para comprometerse con las metas propias del colectivo, cada profesional debe asumir la responsabilidad del éxito o fracaso que le corresponda en el proceso y apoyar de forma constructiva los desacuerdos y procesos de deliberación entre colegas y participantes. Mattessich \& Monsey 
(según citado en Bronstein, 2003) plantean que las metas propias del colectivo incluyen metas viables y claramente definidas, con una visión compartida, una misión, objetivos y estrategias en la que todas y todos las y los participantes estén de acuerdo; compromiso en la toma de decisiones; y colaboradores, colaboradoras con capacidad de compromiso. Billups (según citado en Bronstein, 2003) hace énfasis en dos subprocesos de la colaboración que están estrechamente relacionados con las metas propias del colectivo: identificar y ponderara los intereses, necesidades ó problemas a ser atendidos, establecimiento de metas y desarrollo de planes de acción; además de la negociación e implantación del plan de acción al igual que el compromiso necesario en el seguimiento necesario.

La 'reflexión sobre el proceso' se refiere a la atención que deben poner las colaboradoras y los colaboradores a su proceso de trabajo en conjunto. Esto incluye lo que piensa y reflexiona de forma crítica cada colaborador o colaboradora sobre su relación y proceso de trabajo integrativo para incorporación este análisis a manera de retroalimentación para fortalecer, las relaciones de colaboración y su efectividad al atender situaciones de forma interdisciplinaria.

\section{El Componente de las Prácticas Profesionales de Estudiantes en CAUCE: Una experiencia práctica - académica interdisciplinaria}

Bajo la Ley Núm. 75 del 5 de julio de 1995, la Junta de Planificación elaboró el Plan de Desarrollo Integral y Rehabilitación para la Zona de Planificación Especial de Río Piedras. Esta zona comprende los sectores residenciales de Capetillo, Santa Rita, García Ubarri, Mora y Blondet, además de los sectores comerciales de Paseo de Diego, Plaza de Recreo/Parque de Convalecencia y las calles aledañas. En 1999, mediante la Ley 236, la Legislatura delegó la implantación del Plan de Desarrollo a la Universidad de Puerto Rico, Recinto de Río Piedras y en el 2001 iniciaron sus trabajos el Cuerpo Consultivo para el Desarrollo de Río Piedras y el Grupo de Trabajo Interagencial que preside la Rectora del Recinto de Río Piedras de la Universidad de Puerto Rico. CAUCE abrió sus puertas en 2003 comenzando a revitalización del centro urbano de Río Piedras mediante la coordinación de esfuerzos entre la comunidad y las agencias del gobierno. El mismo es coordinado por el Recinto de Río Piedras, en 
consulta con residentes y comerciantes de esa zona, el gobierno estatal y el Municipio de San Juan. Su trabajo se basa en la unión de fuerzas de la comunidad, la coordinación con las agencias del gobierno y la afirmación de las cualidades de Río Piedras como una verdadera ciudad.

Entre los muchos propósitos que tiene esta Ley 75 de 1995, según enmendada, se destacan los siguientes:

- Promover la rehabilitación física, económica y social de las comunidades y vecindario

- Retener y aumentar la población residente

- Ampliar y mejorar la provisión de servicios a residentes y usuarios del área

- Fortalecer la actividad económica

- Crear un ambiente urbano de usos varios y coherentes (CAUCE).

Es la misión de CAUCE el fomentar alianzas entre los residentes, empresarios, la universidad y el gobierno para fortalecer el tejido social que promueva el desarrollo sustentable del centro urbano de Río Piedras. Siendo esta misión una congruente con los valores de la misión de la Facultad de Ciencias Sociales del Recinto de Río Piedras de la Universidad de Puerto Rico, la cual enfatiza en la preparación y capacitación de profesionales de ayuda para nuestra sociedad, comprometidos con los valores de justicia y transformación social analizando la realidad social mediante la herramienta básica de la investigación social sistemática, se convoca a profesores de varias disciplinas de la Facultad de Ciencias Sociales comprometidos con la producción intelectual de la Universidad de Puerto Rico en la gestión comunitaria, para constituir de forma inicial un equipo de trabajo que se dispuesto a colaborar con la misión de CAUCE y las metas establecidas en el Plan de Desarrollo Integral y Rehabilitación de Río Piedras. El análisis realizado por el equipo de trabajo, nos llevó a concluir que la necesidad de atender el deterioro físico y social del centro urbano tradicional de Río Piedras requería la acción afirmativa de un esfuerzo colectivo participativo de muchos sectores e interdisciplinario (Martínez, 2006). 
Como partes de ese esfuerzo colectivo, se crea el componente de Prácticas Profesionales de Estudiantes en el Centro de acción Urbana Empresarial y Comunitaria (CAUCE) con la participación de profesoras y profesores además de las prácticas profesionales de estudiantes de varios programas sub - graduados y graduados de diferentes disciplinas. Los programas sub - graduados fueron el Departamento de Trabajo Social y el de Psicología Social Comunitaria. Los programas graduados fueron: la Escuela Graduada de Trabajo Social Beatriz Lasalle, Psicología Social Comunitaria y Consejería en Rehabilitación. Participan además, la Clínica de Asistencia Legal de la Facultad de Derecho, docentes y estudiantes de la Escuela Graduada de Ciencias y Tecnología de la Información, además de profesoras y estudiantes de la Facultad de Educación. Los estudiantes practicantes se convierten en los ojos, oídos y brazos de CAUCE en el casco urbano de Río Piedras, especialmente en la comunidad de Capetillo. Los resultados de las experiencias vividas durante los años académicos que trabajé allí han contribuido a reafirmar y validar el modelo de colaboración interdisciplinaria de Bronstein (2003). Desde este modelo y como ente activo dentro del Plan de Trabajo del Proyecto de Río Piedras para el año 2004-2005, aprobado por la Asamblea Comunitaria de Río Piedras en marzo de 2004, el componente de Prácticas profesionales y Clínica de Asistencia Legal participa con el grupo de trabajo de Desarrollo Social y Educación que tiene entre sus metas: lograr una ciudadanía con un alto nivel de educación y de participación democrática en las decisiones sociales que afectan su calidad de vida; un amplio desarrollo organizativo y de autogestión comunitaria; líderes de base formados desde temprana edad; una militancia cotidiana en pro de los derechos de los desposeídos y marginados; y un manejo sensitivo de los conflictos y diferencias que se asocian con la diversidad cultural y étnica de Río Piedras. A partir de esa fecha, años las tareas del equipo interdisciplinario se han concentrado en:

- fortalecer el desarrollo organizativo de los residentes en Capetillo mediante procesos de autogestión comunitaria y la acción social 
- estudios en torno a las necesidades sociales y educativas en la comunidad de Río Piedras, organización y formación de grupos de jóvenes líderes en Capetillo,

- atención a situaciones particulares en las escuelas del Proyecto Colaborativo: Alianza entre la Universidad y las Escuelas vecinas de Río Piedras de la Facultad de Educación. Las escuelas participantes fueron: Luis Muñoz Rivera, nivel elemental, José Celso Barbosa, nivel intermedio y Ramón Vila Mayo, nivel superior. Cabe destacar que en estas escuelas se integran prácticas profesionales de Psicología escolar y de Consejería en Rehabilitación,

- procesos de sistematización de la práctica de trabajo social graduado y psicología social

- Tertulias y talleres educativos que fomentan el desarrollo de líderes, la participación democrática, la convivencia sana, la integración de diferentes sectores de Río Piedras, el conocimiento sobre temas legales y la capacidad de autogestión ( Martínez, 2006).

Las Prácticas Profesionales Estudiantiles han concentrado la mayor parte de sus esfuerzos al apoyo técnico y social en la comunidad de Capetillo, específicamente en el sector identificado como la Quebrada Juan Méndez, un sector inundable rescatado originalmente en los años '30. La Quebrada Juan Méndez, recordada por algunos como la Isla de Diablo, es un sector de marginación social en una zona urbana de la ciudad capital. A partir de agosto de 2003, se inició una colaboración entre los departamentos de Trabajo Social (subgraduado) y Psicología (subgraduado y graduado), la Escuela Graduada de Trabajo Social Beatriz Lassalle y la Clínica de Asistencia Legal de la Escuela de Derecho, solicitada por líderes comunitarios del sector, con el propósito de brindar orientación y apoyo a 22 familias inmersas en un proceso de realojo temporal ante la proximidad de la construcción de un complejo de viviendas en un área no inundable cercana a la Quebrada Juan Méndez, como parte de la agenda existente para la revitalización del caso urbano de Río Piedras. 
La interdisciplinariedad y la interdependencia del componente de las prácticas profesionales de estudiantes se ejercieron de manera cabal en este proceso al insertarse en el sector con una actitud de solidaridad colectiva demostrada en las múltiples visitas, entrevistas, reuniones del equipo integrativo de las diferentes disciplinas con los y las residentes. Las actividades realizadas en cada disciplina giraban en tormo a las 'metas y objetivos propios del colectivo' formulados en consenso luego de las ponderaciones realizadas y analizadas por el grupo en conjunto con la participación de la comunidad.

\section{Figura 2 \\ Ejemplos de Metas Propias del colectivo en el Plan de Trabajo Interdisciplinario: Componente de Prácticas Profesionales de Estudiantes en CAUCE}

\begin{tabular}{|c|c|c|}
\hline \multicolumn{3}{|c|}{$\begin{array}{l}\text { Meta \#1: Promover el desarrollo de actividades dirigidas al mejoramiento de las } \\
\text { condiciones de vida de los residentes del casco urbano de Río Piedras, tales como } \\
\text { la salud, la recreación, la educación, el desarrollo económico y el desarrollo de } \\
\text { vivienda, fomentando la organización y la participación comunitaria }\end{array}$} \\
\hline Objetivos & Personas responsables & Indicadores de éxito \\
\hline $\begin{array}{l}\text { 1.1. Continuar } \\
\text { ofreciendo apoyo técnico } \\
\text { y social, además del } \\
\text { seguimiento pertinente al } \\
\text { sector Quebrada Juan } \\
\text { Méndez en el desarrollo } \\
\text { de los procesos de } \\
\text { construcción de } \\
\text { viviendas para sus } \\
\text { residentes, de realojo de } \\
\text { dichos residentes, } \\
\text { además del rescate del }\end{array}$ & $\begin{array}{l}\text { Estudiantes de Maestría } \\
\text { practicante de Psicología } \\
\text { Social Comunitaria } \\
\text { Trabajadores Sociales en } \\
\text { Adiestramiento: } \\
\text { Bachillerato y Maestría } \\
\text { Clínica de Asistencia } \\
\text { Legal } \\
\text { Prof. Dolores Miranda } \\
\text { Prof. Ana M. Martínez } \\
\text { Prof. Magda Orfila } \\
\text { Lic. Mirta Morales }\end{array}$ & $\begin{array}{l}\text { 1.1. Evaluación } \\
\text { cualitativa interna sobre } \\
\text { la experiencia del apoyo } \\
\text { técnico y social, además } \\
\text { del seguimiento recibido } \\
\text { con relación a los } \\
\text { procesos de construcción } \\
\text { de viviendas y realojo de } \\
\text { los residentes por las } \\
\text { diferentes disciplinas a } \\
\text { diciembre de } 2005 \text {. }\end{array}$ \\
\hline $\begin{array}{l}\text { 1.2 Continuar Proyecto } \\
\text { de Alfabetización } \\
\text { iniciado el pasado } \\
\text { semestre. }\end{array}$ & $\begin{array}{l}\text { Estudiantes de Trabajo } \\
\text { Social y Psicología } \\
\text { Social } \\
\text { Com. Adiestrados por } \\
\text { Ana Baca, estudiante } \\
\text { graduada Psicología y } \\
\text { Prof. Teresa Pujols, } \\
\text { Facultad de Educación }\end{array}$ & $\begin{array}{l}1.2 \text { Número de personas } \\
\text { participantes en el } \\
\text { Proyecto } \\
\text { Sistematización de la } \\
\text { experiencia / entrevistas } \\
\text { a participantes }\end{array}$ \\
\hline
\end{tabular}




\begin{tabular}{|c|c|c|}
\hline Objetivos & Personas responsables & Indicadores de éxito \\
\hline $\begin{array}{l}3.1 \text { Ofrecer talleres sobre } \\
\text { la tolerancia y manejo de } \\
\text { la diversidad cultural } \\
\text { como herramienta para } \\
\text { la convivencia sana en } \\
\text { trabajo directo con la } \\
\text { comunidad dominicana } \\
\text { en Río Piedras. }\end{array}$ & $\begin{array}{l}\text { Estudiantes de } \\
\text { Psicología social } \\
\text { Prof. Ruth Nina Estrella } \\
\text { Estudiantes de Maestría } \\
\text { Practicante de Psicología } \\
\text { Social Comunitaria } \\
\text { Trabajadores Sociales en } \\
\text { Adiestramiento: } \\
\text { Bachillerato y Maestría } \\
\text { Estudiante Escuela } \\
\text { Graduada de Consejería } \\
\text { en Rehabilitación } \\
\text { Clínica de Asistencia } \\
\text { legal } \\
\text { Supervisores de las } \\
\text { diversas Prácticas } \\
\text { Profesionales }\end{array}$ & $\begin{array}{l}\text { 3.1 Evaluación } \\
\text { cualitativa interna y } \\
\text { externa sobre la } \\
\text { experiencia del apoyo y } \\
\text { colaboración al grupo } \\
\text { Joluk, a diciembre de } \\
2005 \text {. } \\
\text { 3.2.1. Número de grupos } \\
\text { organizados / Registro } \\
\text { de participantes. } \\
\text { 3.2.2. Evaluación } \\
\text { participativa de las } \\
\text { experiencias, a } \\
\text { diciembre de } 2005 \text {. }\end{array}$ \\
\hline
\end{tabular}

Las actividades estaban dirigidas a facilitar, orientar y educar sobre el proceso. Todas y todos los estudiantes participantes asistieron a un taller, previo a comenzar sus tareas en la comunidad, de orientación sobre el proceso de intervención en las visitas y entrevistas de desahogo a realizar sobre un tema tan sensitivo como puede ser el de un realojo de residencia. Este taller fue preparado y ofrecido para todos los estudiantes por la directora del programa de Psicología del Recinto. Una visita a uno de los hogares de residentes podía realizarse con una pareja de estudiantes de Trabajo Social y de Psicología Social Comunitaria, ya fuera de los programas sub graduado y graduado, utilizando un mismo formato que se diseño con la colaboración de las disciplinas de Trabajo Social, Psicología Social Comunitaria y el asesoramiento de los estudiantes de la Clínica de Asistencia Legal de la Escuela de Derecho. Este es un ejemplo de uno de los componentes básicos y constante en un modelo de colaboración interdisciplinaria, 'las actividades de nueva creación', cuyo alcance colectivo logra 
mucha más magnitud que si se diera de manera individual. Las reuniones del grupo integrativo dos veces al mes (o con más frecuencia cuando era necesario), al igual que las reuniones de pares cada semana para discutir, deliberar sobre situaciones relevantes ocurridas durante el proceso de orientación, discusión y negociación sobre el realojo, garantizaban la 'flexibilidad' en la aplicación a la vez que promovía la 'reflexión sobre el proceso', integrando este análisis crítico al fortalecimiento del propio equipo integrativo, al Proyecto CAUCE y a las relaciones de colaboración con la comunidad para de esta manera alcanzar las metas propuestas.

\section{Conclusión y Reflexión final}

Pensar que la interdisciplinariedad puede practicarse profesionalmente, ya sea en las experiencias de las prácticas profesionales universitarias de nuestros y nuestras estudiantes, así como en sus posteriores escenarios profesionales de trabajo sin que se haga el mismo ejercicio desde su formación universitaria como parte del proceso de enseñanza -aprendizaje es una falacia. Es necesario examinar desde el interior de cada facultad la capacidad de integración y colaboración que se está dando o promoviendo con otras facultades para el análisis de la realidad de la sociedad como una totalidad $\mathrm{y}$ no como una fragmentada, utilizando como justificación la idea negativa que el cruzar las fronteras de la disciplina implica perder los parámetros filosóficos de cada disciplina. Ante tal disyuntiva Calderón, Núñez \& Serrano (2003) postulan como primera necesidad, la convergencia de ciertos problemas generales que están muy relacionados con aspectos centrales de las ciencias de la vida. Nos indican que para resolver esos problemas hay que recurrir a nociones cardinales que recubren mecanismos comunes ya que es el estudio de esos mecanismos comunes lo que exige y exigirá cada vez más el trabajo interdisciplinario. Estos problemas comunes se enfocan en la transformación, la búsqueda del equilibrio y el intercambio.

Se hace necesario el ejercicio académico del trabajo interdisciplinario, el esfuerzo de que cada disciplina participante se oriente a alcanzar una visión integral de los intereses, necesidades y problemas que atendemos cotidianamente con una comprensión más crítica de la 
realidad que definitivamente se oriente las formas de acción y trabajo integrativo a la transformación social. Estoy convencida en que fomentar la creación de espacios de intercambio de saberes interdisciplinarios entre las estudiantes y los estudiantes de este recinto universitario es una respuesta adecuada al desfase entre la educación superior, la investigación, las competencias profesionales requeridas ante los retos y desafíos actuales que plantea el mundo profesional urbano. Estoy convencida de que la interdisciplinariedad en la formación del egresado de la Universidad de Puerto Rico, contribuirá a la formación de una profesional, de un profesional disciplinada, disciplinado en el aprendizaje, con capacidad de reflexión, análisis crítico, destrezas para la investigación científica, la acción social transformadora, entendimiento comprensivo de su entorno y solidaridad hacia sus semejantes. 


\section{Referencias}

Ander-Egg, Ezequiel. (1999). Interdisciplinariedad en Educación. Buenos Aires: Magisterio del Río de la Plata.

Bronstein, Laura. (2003). A model for interdisciplinary collaboration. Social Work, 48, (3), 297-306.

Calderón Soto, Jaime, Nuñez Cruz, Junior A. \& Serrano García, Irma. (2003). Una reflexión sobre la educación interdisciplinaria: el caso de PAIVS. En Europa Piñero, Lilliana Ramos \& Pedro Subirats ( Eds.). Congreso de Investigación sobre la Universidad (pp. $151-165$ ).

Ley Especial para la Rehabilitación de Río Piedras. Ley Núm. 75 del 5 de julio de 1995.

Martínez Vizcarrondo, Ana Maritza. (2006). Universidad y su entorno riopedrense: Historias de un esfuerzo comunitario. En Alma I. Acosta Cartagena, Edda L. Arzola Rivera y Europa Piñero

González. (Eds.), Universidad Centenaria: ¿Y Ahora Qué?, 131-136. San Juan: Asociación Puertorriqueña de Profesores Universitarios (APPU).

Parker, Debra. (2005). Examining variables related to successful collaboration on the hospice team. Health Social Work, 30, (4), $279-286$.

Romero Sánchez, Patricia. (2005). La práctica interdisciplinaria en la conciliación psicojurídica. Revista Pericia, 1, (1). Recuperado el 15 de octubre de 2008, de http://www.idrc.ca/lacro/ev102764201-DO_TOPIC.html 
United Nations Educational, Scientific and Cultural Organization. (2000). Proyecto Profesionales de la Ciudad. Programa de Gestión de Transformaciones Sociales de las Ciencias Sociales y Humanas (MOST). Recuperado el 9 de septiembre de 2008, de mhtml:file//E:IUNESCO Proyecto Profesionales de la ciudad (3) $\backslash$ MOST - Profesionales de la ciudad.

Universidad de Puerto Rico. (2006). Diez para la década: Agenda para la planificación 2006-2016. Puerto Rico: Oficina del Presidente. 
